\title{
Laparoscopic gastrectomy for advanced cancer: a technical challenge
}

\author{
TAKASHi Aiko \\ First Department of Surgery, Kagoshima University School of Medicine, 8-35-1 Sakuragaoka, Kagoshima 890-0075, Japan
}

Laparoscopic surgery has been employed for various solid tumors such as colorectal and ovarian cancers. Gastric cancer can also be a target of this technique and the tumors at early stages have been treated by laparoscopic wedge resection or partial distal gastrectomy [1,2]. In this issue of Gastric Cancer, Uyama et al. [3] report their experience of treating advanced gastric cancer with total gastrectomy and pancreaticosplenectomy (PS) with D2 lymphadenectomy. Their article will have a definite clinical impact in terms of technical achievement, and their pioneering spirit should be admired. It is now evident that almost all types of gastrectomies could be performed by laparoscopic techniques. The next question will be who would benefit from this procedure. There are, however, some problems to be solved before the question is answered.

Uyama et al. [3] emphasize that this procedure has several advantages over open surgery, such as minimal postoperative pain, quicker mobilization, and better cosmetic results. In cancer treatment, however, these features can be regarded as advantages only when curability comparable to that in open surgery is guaranteed. In this sense, early gastric cancer is a good target for laparoscopic gastrectomy because the nodal metastasis is rather limited and the serosal surface is intact. In advanced gastric cancers, however, it has traditionally been believed, at least in Japan, that en-bloc resection with wide lymphadenectomy gives oncologically better results. Gentle manipulation of intraabdominal organs may also be important to prevent scattering of cancer cells. I wonder whether laparoscopic gastrectomy can assure these results.

The problem of $\mathrm{CO}_{2}$ pneumoperitoneum should also be taken into consideration. Recent studies on the adverse effects of laparoscopic procedures suggest that $\mathrm{CO}_{2}$ pneumoperitoneum may influence peritoneal defense mechanisms and accelerate tumor growth and adherence to the peritoneum [4-6]. Port-site recurrence is a well known example. Although this risk may be very small [7], it should be considered, particularly when the tumor is exposed to the peritoneal surface.

If open and laparoscopic gastrectomies are equally safe and effective, we should consider costeffectiveness. The performance of laparoscopic gastrectomy obviously has a steep learning curve and, even with experienced surgeons such as Uyama and colleagues, this operation took more than $10 \mathrm{~h}$. Six trocars were placed, and the left lower wound was increased to $4 \mathrm{~cm}$ in length at the end of the operation to remove the dissected organs. After the laparoscopic procedure, patients may require doses of analgesics similar to those required after open surgery. One of the issues that the authors seem to have overlooked is the possible formation of a pancreatic fistula after laparoscopic PS. A pancreatic fistula, a not uncommon complication after gastrectomy, develops in about $20 \%$ of patients who have had open-surgical PS and prolongs their hospital stay. If laparoscopic PS were to be associated with an increased risk of fistula formation, its cost-effectiveness would be greatly reduced.

The final problem is the feasibility of this technique as a routine procedure. The authors must have made great efforts to develop this operation. Obviously, the techniques employed are not simple and are not easy to learn. The outcomes of the operation will be directly related to the volume of the surgeons' experience. The establishment of teaching programs in some specialized centers may be required.

I believe that, for the time being, this operation should be reserved for selected patients and experienced surgeons. The candidate patients should be fully informed of the possible problems, mentioned above, as well as the expected benefits. The pitfalls of the technique and the patterns of the patients' postoperative courses will become clear as the number of patients increases. It seems likely that laparoscopic gastrectomy will play a greater role in the future, especially for tumors at early stages, and I hope that the procedures 
will progress on the basis of careful oncological studies. A randomized trial comparing totally laparoscopic versus open gastrectomy may be necessary in the future to provide definitive answers to the clinical and biological questions raised by this technique.

\section{References}

1. Ohgami M, Otani Y, Kumai K, Kubota T, Kim YI, Kitajima M. Curative laparoscopic surgery for early gastric cancer: 5 years experience. World J Surg 1999;23:187-93.

2. Shiraishi N, Adachi Y, Kitano S, et al. Indication for and outcome of laparoscopy-assisted Billroth I gastrectomy. Br J Surg 1999;86: $541-4$.
3. Uyama I, Sugioka A, Fujita J, Komori Y, Matsui H, Hasumi A. Laparoscopic total gastrectomy with distal pancreatosplenectomy and D2 lymphadenectomy for advanced gastric cancer. Gastric Cancer 2000;2:230-4.

4. Volz J, Kolster S, Spacek Z, Paweletz N. The influence of pneumoperitoneum used in laparoscopic surgery on an intraabdominal tumor growth. Cancer 1999;86:770-4.

5. Dorrance HR, Oien K, O'Dwyer PJ. Effects of laparoscopy on intraperitoneal tumor growth and distant metastases in an animal model. Surgery 1999;126:35-40.

6. Neuhaus SJ, Watson DI, Ellis T, Rowland R, Rofe AM, Pike GK, et al. Wound metastasis after laparoscopy with different insufflation gases. Surgery 1998;123:579-83.

7. Pearlstone DB, Feig BW, Mansfield PF. Port site recurrences after laparoscopy for malignant disease. Semin Surg Oncol 1999;16: $307-12$. 